\title{
Distribution of Interacting Ionic Particles in Disordered Media
}

\author{
Ramin Golestanian \\ Institute for Advanced Studies in Basic Sciences, Zanjan 45195-159, Iran \\ and Institute for Studies in Theoretical Physics and Mathematics, P.O. Box 19395-5531, Tehran, Iran
}

(November 8, 2018)

\begin{abstract}
Equilibrium distribution of interacting ionic particles in a charged disordered background is studied using the nonlinear Poisson-Boltzmann equation. For an arbitrarily given realization of the disorder, an exact solution of the equation is obtained in one dimension using a mapping of the nonlinear Poisson-Boltzmann equation to a self-consistent Schrödinger equation. The resulting density profile shows that the ions are delocalized, despite what the equivalent Schrödinger formulation in one dimension would suggest. It is shown that the ions are not distributed so as to locally neutralize the background, presumably due to their mutual interactions.
\end{abstract}

Dynamics of interacting charged particles in a heterogeneous Coulombic background, which is a medium with a quenched distribution of charge centers, is a complex phenomenon involving long equilibration times, a multitude of time scales and various intermediate regimes, and competing mechanisms 近田. All these complications, which are primarily due to the long-range nature of Coulomb interaction, have so far made it extremely difficult for even rather sophisticated simulation techniques to provide us with a conclusive picture [4,5].

Perhaps a most preliminary step is to determine whether the equilibrium distribution of the mobile ions corresponds to a localized or an extended state, in analogy to the quantum mechanical (QM) problem of disordered electronic systems [6]. The answer to this question is determined by a delicate balance between the following competing effects: (i) attraction of the mobile ions to the opposite fixed charges and the tendency towards local neutralization, (ii) repulsion between similarly charged mobile ions, and (iii) entropy of the mobile ions. (Note that for simplicity of the argument we have assumed that all the mobile ions are similarly charged whereas all the fixed charges are of the opposite type.) While attraction tends to localize the mobile ions in the neighborhood of the charge centers, repulsion and entropy both favor delocalization. One can imagine that determining the outcome of this balance in simulations could be a very difficult task due to the progressively long escape times for the deep Coulomb potential wells. On the other hand, it is also not clear if one can trust approximate theoretical schemes, which may artificially weaken or strengthen any of the above tendencies, in making feasible predictions to this end.

For example, it has been shown that if we neglect the mutual repulsion between the mobile ions, the answer to the above question depends on the dimensionality [3,5, 4, 8]. One can show that the disorder-averaged density-density correlation function for the mobile ions behaves as $\overline{\langle c(\mathbf{0}) c(\mathbf{x})\rangle} \sim \exp \left[-(x / \xi)^{2-d}\right]$ in $d$ dimensions [9], and therefore the ions are localized for $d<$
2 (with $\xi$ being the localization length). On general grounds one might then expect that the interaction between the mobile ions could be effectively taken into account by introducing the notion of screening, which would change the density-density correlation function to $\overline{\langle c(\mathbf{0}) c(\mathbf{x})\rangle} \sim \exp \left[-(x / \xi)^{2-d} g(\kappa x)\right]$, where $g$ is an exponentially decaying function and $\kappa^{-1}$ is the corresponding Debye screening length that is related to the density of the mobile ions. The appearance of the exponential damping now controls the divergence for $d<2$, and consequently the mobiles ions will be delocalized in any dimension. The simplistic implementation of screening using Debye-Hückel theory, however, is based on a linearized approximation of the nonlinear PoissonBoltzmann (PB) theory, which is known to break down in the vicinity of the charge centers where the mobile ions could presumably be trapped for relatively long times. Therefore, it seems that a reliable answer to the localization question can only be obtained through an exact treatment of the full nonlinear PB formulation. This is what I attempt to do in this article.

I consider a one dimensional medium with a quenched distribution of charge centers and a finite density of oppositely charged mobile ions, and show that one can map the corresponding nonlinear PB equation to a selfconsistent Schrödinger equation. An exact solution of the resulting equation is then presented for an arbitrary realization of the disorder. The resulting density profile shows that the ions are in an extended state. It is shown that the mutual interactions of the mobile ions could lead to a gross violation of the anticipated local neutrality.

Consider a set of fixed negatively charged ions described by the given charge density $\rho_{\mathrm{f}}(\mathbf{x})$, embedded in a cloud of positively charged mobile ions with concentration $c(\mathbf{x}, t)$. The electric potential $\phi(\mathbf{x}, \mathrm{t})$ is then given by the solution of the Poisson equation:

$$
-\nabla^{2} \phi=\frac{4 \pi}{\epsilon}\left(e c+\rho_{\mathrm{f}}\right),
$$

in which $\epsilon$ is the dielectric constant of the background, 
and $e$ is the electron charge. The dynamics of $c(\mathbf{x}, t)$ is governed by a conservation law of the form $\partial_{t} c+\nabla \cdot \mathbf{J}=0$, in which $\mathbf{J}=-D \nabla c+c \mathbf{v}$ is the current density, where $\mathbf{v}(\mathbf{x}, t)$ is the velocity field corresponding to the mobile ions, and $D$ is their diffusion constant. In a mean-field approximation, the velocity field of the ions at each point is determined by the local value of the electric field $\mathbf{E}(\mathbf{x}, t)$ as $\mathbf{v}=\mu \mathbf{E}=-\mu \nabla \phi$, where $\mu$ is the electric mobility of the ions. Using the self-consistency relation, the continuity equation can be written as

$$
\partial_{t} c=D \nabla^{2} c+\mu \nabla \cdot(c \nabla \phi)
$$

The above two equations describe the nonlinear dynamics of mobile ions in the presence of a given distribution of fixed ions [10,11. At equilibrium we have $\partial_{t} c=0$, and Eq.(2) yields $c \sim \exp (-\mu \phi / D)$, where equilibration at a temperature $T$ implies an Einstein relation $D=(\mu / e) k_{B} T$. Inserting the Boltzmann form for $c$ back into the Poisson equation then yields the PB equation.

Let us now restrict ourselves to the one dimensional case where the equations prove to be more tractable. Eliminating the concentration field $c$ from the two equations, one obtains an equation for the electrostatic potential, which can be integrated twice to yield

$$
\begin{aligned}
\partial_{t} \phi(x, t)- & \partial_{t} \phi(-\infty, t)=D \partial_{x}^{2} \phi+\frac{\mu}{2}\left(\partial_{x} \phi\right)^{2} \\
+ & \frac{4 \pi}{\epsilon}\left[\mu \int_{-\infty}^{x} d x^{\prime} \rho_{\mathrm{f}}\left(x^{\prime}\right) \partial_{x} \phi\left(x^{\prime}\right)+D \rho_{\mathrm{f}}(x)\right]
\end{aligned}
$$

where the boundary condition $E( \pm \infty, t)=0$ has been implemented. The above equation can be called a dynamical $\mathrm{PB}$ equation [12].

We focus, for simplicity, on the specific example of a series of $N+1$ negatively charged permeable plates with charge (number) density $\sigma$ and surface area $A$, which are placed at random locations $x_{n}$ on the $x$-axis, i.e., $\rho_{\mathrm{f}}(x)=-e \sigma \sum_{n=0}^{N} \delta\left(x-x_{n}\right)$.

Using a so-called Cole-Hopf transformation $\phi(x, t)-$ $\phi(-\infty, t)=(2 D / \mu) \ln W(x, t)$, Eq. (3) can be written as a Schrödinger-like equation for $W(x, t)$ :

$$
\begin{aligned}
\partial_{t} W(x, t) & =D \partial_{x}^{2} W(x, t) \\
& +\frac{2 D}{\lambda} \sum_{n=0}^{N}\left[\varepsilon_{n}(t) \Theta\left(x-x_{n}\right)-\delta\left(x-x_{n}\right)\right] W(x, t)
\end{aligned}
$$

where

$$
\varepsilon_{n}(t)=-\left(\frac{\partial_{x} W\left(x_{n}^{+}, t\right)+\partial_{x} W\left(x_{n}^{-}, t\right)}{W\left(x_{n}, t\right)}\right),
$$

and $\lambda \equiv \epsilon D /(\pi e \sigma \mu)=\epsilon k_{B} T /\left(\pi e^{2} \sigma\right)$ is a characteristic length scale corresponding to the relative strength of the electrostatic and thermal interactions. Note that Eq.(4) is still nonlinear due to the self-consistent coupling of the so-called asymmetry fields $\varepsilon_{n}(t)=\mu E\left(x_{n}, t\right) / D$.

The value of the asymmetry field at each point can be calculated from Gauss' law as

$$
\varepsilon_{n}(t)=\frac{2}{\lambda}\left(\frac{2 Q_{n}}{e \sigma A}\right)
$$

in which $Q_{n}$ is the net amount of (uncompensated) charge on the left hand side of point $x_{n}$. (Overall neutrality then implies that $-Q_{n}$ should be the net amount of charge on the right hand side of point $x_{n}$.) In other words, $\varepsilon_{n}(t)$ measures the relative deviation from local neutrality as seen at $x_{n}$.

The dynamics described by Eq.(14) is difficult due to the time dependence of the $\varepsilon_{n}(t)$ fields. We thus focus on the long time limit of the dynamics, when the ions are expected to equilibrate in the disordered medium. In this limit, the asymmetry fields are time independent, and the Schrödinger equation reads

$$
-\frac{\lambda}{2} \partial_{x}^{2} W(x)+V(x) W(x)=0,
$$

where the effective potential is given as

$$
V(x)=\sum_{n=0}^{N}\left[-\varepsilon_{n} \Theta\left(x-x_{n}\right)+\delta\left(x-x_{n}\right)\right] .
$$

Note the peculiar feature that the delta function peaks in the potential, which correspond to the attractive sites for the mobile ions, appear as effective repulsive potential wells in the equivalent QM description!

To complete the QM formulation, we need a normalization for $W(x)$. Using the equilibrium relation $c \sim \exp (-\mu \phi / D)$, and the definition of $W$, we can relate $c$ to $W$ as $c(x) \sim 1 / W^{2}(x)$. Conservation of the overall number of mobile ions can thus be written as

$$
\frac{1}{L} \int_{-L / 2}^{L / 2} d x\left[\frac{1}{W(x)}\right]^{2}=1
$$

where $L$ is the system size.

We can now solve the nonlinear self-consistent QM problem described by Eqs.(5), (7), and (9), because the potential is a constant in each interval. The solution of Eq.(7) is a series of plane waves:

$$
W(x)= \begin{cases}A_{0}+B_{0} x, & \text { for } x<x_{0}, \\ A_{j} e^{i k_{j}\left(x-x_{j}\right)} & \\ +B_{j} e^{-i k_{j}\left(x-x_{j}\right)}, & \text { for } x_{j-1}<x<x_{j}, \\ A_{N+1}+B_{N+1} x, & \text { for } x_{N}<x\end{cases}
$$

where

$$
k_{j}=\left[\frac{2}{\lambda} \sum_{n=0}^{j-1} \varepsilon_{n}\right]^{1 / 2}
$$

for $j=1, \cdots, N$. We should then match the neighboring solutions across the boundary points based on the 
following three requirements: (i) $W(x)$ should be continuous, (ii) the jump in $\partial_{x} W / W$ should be set to $2 / \lambda$ as implied by the delta function peaks in $V(x)$, and (iii) the asymmetry fields should satisfy the self-consistency relation of Eq.(5). These criteria translate into a series of self-consistency equations for the wave vectors:

$$
\begin{aligned}
e^{2 i k_{j} \Delta_{j}} & =\left[\frac{1+\frac{\lambda^{2}}{4}\left(k_{j+1}^{2}-k_{j}^{2}\right)-i k_{j} \lambda}{1+\frac{\lambda^{2}}{4}\left(k_{j+1}^{2}-k_{j}^{2}\right)+i k_{j} \lambda}\right] \\
& \times\left[\frac{1+\frac{\lambda^{2}}{4}\left(k_{j-1}^{2}-k_{j}^{2}\right)-i k_{j} \lambda}{1+\frac{\lambda^{2}}{4}\left(k_{j-1}^{2}-k_{j}^{2}\right)+i k_{j} \lambda}\right],
\end{aligned}
$$

for $j=1, \cdots, N$, where $\Delta_{j}=x_{j}-x_{j-1}$ and $k_{0}=k_{N+1}=$ 0 . For any given set of $\Delta_{j} \mathrm{~s}$, one should solve these coupled nonlinear equations to determine the wave vectors. The matching requirements together with the normalization condition of Eq.(9), can then give us the full solution, and thus the density profile of the ions.

In order to determine whether the ions are in an extended state, or localized near the adsorbing sites, we need to find out whether the $k_{j}$ s that satisfy Eq.(12) are real or imaginary. Instead of trying to solve for $k_{j} \mathrm{~s}$ exactly, I shall first resort to approximate schemes. Physically we expect to have a tendency towards localization at low temperatures or for strong disorder, and nearly free ions with uniform distribution at high temperatures or for weak disorder.

Let us first focus on the case of strong disorder where we have $\lambda \ll \Delta_{j}$, and try to solve Eq.(12) perturbatively. I find

$$
\begin{aligned}
k_{j}= & \frac{\pi}{\Delta_{j}}-\frac{2 \pi \lambda}{\Delta_{j}^{2}}+\frac{4 \pi \lambda^{2}}{\Delta_{j}^{3}}-\left(8 \pi-\frac{\pi^{3}}{6}\right) \frac{\lambda^{3}}{\Delta_{j}^{4}} \\
& +\frac{\pi^{3} \lambda}{4 \Delta_{j}^{2}}\left(\frac{\lambda^{2}}{\Delta_{j+1}^{2}}+\frac{\lambda^{2}}{\Delta_{j-1}^{2}}\right)+O\left(\frac{\lambda^{4}}{\Delta^{5}}\right),
\end{aligned}
$$

for the first few terms, which is real. This proves, strictly speaking, that if the ions are localized, the localization length should be greater than $\Delta^{5} / \lambda^{4}$. However, it looks rather unlikely that higher order corrections start to develop imaginary components, which implies that the ions should be delocalized even in the strong disorder limit.

To check this, I have solved Eq.(12) numerically for various choices of $\left\{\Delta_{j}\right\}$, and the results for the values of $\left\{k_{j}\right\}$ were always real. For example, for the case of 11 charged centers with $\Delta_{1} / \lambda=0.1, \Delta_{2} / \lambda=0.5, \Delta_{3} / \lambda=2$, $\Delta_{4} / \lambda=0.8, \Delta_{5} / \lambda=0.01, \Delta_{6} / \lambda=7, \Delta_{7} / \lambda=3.2$, $\Delta_{8} / \lambda=0.3, \Delta_{9} / \lambda=1.6$, and $\Delta_{10} / \lambda=0.0002$, I find $k_{1} \lambda=33.84, k_{2} \lambda=7.33, k_{3} \lambda=1.42, k_{4} \lambda=4.95$, $k_{5} \lambda=316.69, k_{6} \lambda=0.399, k_{7} \lambda=0.757, k_{8} \lambda=12.599$, $k_{9} \lambda=1.933$, and $k_{10} \lambda=15710$, which are all real. This confirms that the ions are in an extended state.

We can also calculate the asymmetry fields from the above results as

$$
\varepsilon_{j}=\frac{\lambda}{2}\left(k_{j+1}^{2}-k_{j}^{2}\right) \simeq \frac{\lambda}{2}\left(\frac{\pi^{2}}{\Delta_{j+1}^{2}}-\frac{\pi^{2}}{\Delta_{j}^{2}}\right),
$$

to the leading order. This shows that unlike what one might expect, the distribution of ions is not predominantly determined by the criterion of local neutralization of the background, which would yield $\varepsilon_{j}=0$.

Now let us address the peculiar features of the formulation, and try to understand them better: (i) Why do the delta function peaks, corresponding to adsorbing sites in the real problem, appear as repulsive in the effective potential $V(x)$ ? (ii) While we expect a Schrödinger equation with random potential in one dimension to lead to localization [6], why do we find that the ions are delocalized? To answer these questions, we should note the unusual normalization condition of Eq.(9) above. A true wave function for this problem should be defined as $\Psi(x)=1 / W(x)$ according to Eq.(9), leading to a corresponding Schrödinger equation as

$$
-\frac{\lambda}{2} \partial_{x}^{2} \Psi(x)+\left[\lambda\left(\frac{\partial_{x} \Psi(x)}{\Psi(x)}\right)^{2}-V(x)\right] \Psi(x)=0,
$$

which is highly nonlinear.

While the sign of the effective potential is now reversed corresponding to attractive delta function peaks, an additional effective repulsive self-consistent potential has also appeared. This potential, which is proportional to the the electrostatic pressure $\sim(\nabla \phi)^{2}$, disfavors pileup of the ions in the vicinity of the attractive sites and promotes delocalization. This extra self-consistent term, which is a direct consequence of the repulsive interactions between the mobile ions, explains the discrepancy between the predictions of this theory and conventional Schrödinger equation in a random potential.

In conclusion, I have presented an exact analysis of the problem of interacting mobile ions in a random background of fixed ions in one dimension. The density profile of the ions at equilibrium is shown to be extended (as opposed to localized) as a consequence of the interactions between the mobile ions. Since the one dimensional problem in the strong disorder limit that has the best chance of leading to localization is proven to result in an extended state, it is plausible to assert that the same result should hold true in general, i.e., in higher dimensionalities and for weaker disorder. Although I have only considered positively charged mobile ions in a negative random background, the formulation can be readily generalized to contain both species of mobile ions and fixed ions.

I am grateful to M.P.A. Fisher, M. Kardar, M. Mezard, and M. Sahimi for invaluable discussions and comments. This research was supported in part by the National Science Foundation under Grant No. DMR-98-05833. 
[1] J. Krager and D.M. Ruthven, Diffusion in Zeolites and Other Microporous Solids (Wiley, New York, 1992).

[2] J.-P. Bouchaud and A. Georges, Phys. Rep. C 195, 127 (1990).

[3] M.W. Deem and D. Chandler, J. Stat. Phys. 76, 911 (1994).

[4] A.R. Mehrabi and M. Sahimi, Phys. Rev. Lett. 82, 735 (1999).

[5] M.W. Deem, Phys. Rev. Lett. 83, 1694 (1999); M. Sahimi and A.R. Mehrabi, Phys. Rev. Lett. 83, 1695 (1999).

[6] See e.g.: D.J. Thouless, Phys. Rep. 13, 93 (1974); P.A. Lee and T.V. Ramakrishnan, Rev. Mod. Phys. 57, 287 (1985).

[7] A.K. Chakraborty, D. Bratko, and D. Chandler, J. Chem. Phys. 100, 1528 (1994).
[8] J.-M. Park and M.W. Deem, Phys. Rev. E 58, 1487 (1998).

[9] I am grateful to Prof. M.P.A. Fisher for pointing this out to me.

[10] V.G. Levich, Physicochemical Hydrodynamics (PrenticeHall, London, 1962).

[11] R. Golestanian, Europhys. Lett. 52, 47 (2000).

[12] Eq.(3) belongs to the general class of the celebrated KPZ equations (M. Kardar, G. Parisi, and Y.C. Zhang, Phys. Rev. Lett. 56, 889 (1986)), with the coupling to the source appearing both in the additive and multiplicative forms. However, note that in this context the sources represent quenched degrees of freedom, which makes it somewhat different from the standard KPZ problems. 